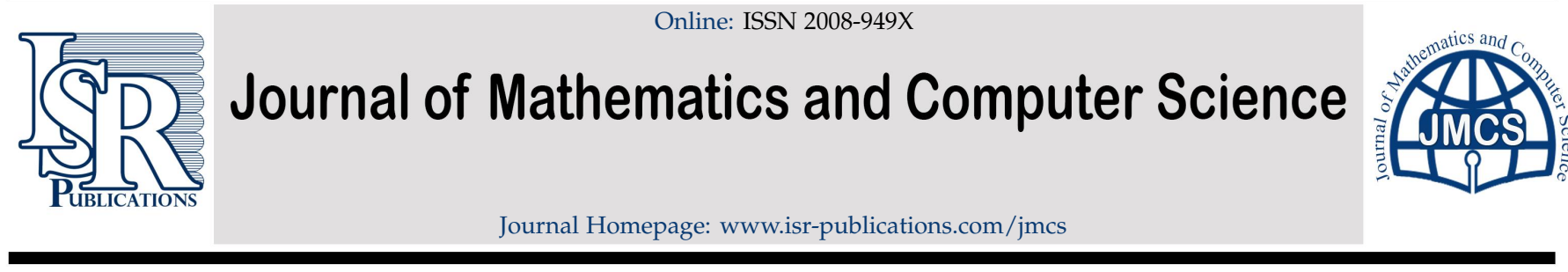

\title{
On unified Gould-Hopper based Apostol-type polynomials
}

\author{
Waseem Ahmad Khan ${ }^{a}$, Kottakkaran Sooppy Nisar ${ }^{b, *}$, Mehmet Acikgoz ${ }^{\mathrm{c}}$, Ugur Duran ${ }^{\mathrm{d}}$, Abdallah Hassan \\ Abusufian $^{b}$ \\ ${ }^{a}$ Department of Mathematics and Natural Sciences, Prince Mohammad Bin Fahd University, P. O. Box 1664, Al Khobar 31952, Saudi \\ Arabia. \\ ${ }^{b}$ Department of Mathematics, College of Arts and Sciences, Wadi Aldawaser, Prince Sattam bin Abdulaziz University, Saudi Arabia. \\ ${ }^{c}$ Department of Mathematics, Faculty of Arts and Sciences, University of Gaziantep, TR-27310 Gaziantep, Turkey. \\ ${ }^{d}$ Department of the Basic Concepts of Engineering, Faculty of Engineering and Natural Sciences, Iskenderun Technical University, \\ TR-31200 Hatay, Turkey.
}

\begin{abstract}
In this paper, we consider unified Gould-Hopper based Apostol-type polynomials and investigate some of their formulas including several implicit summation formulae and some symmetric identities by the series manipulation method. Moreover, we acquire several new results for unified Gould-Hopper based Apostol-type polynomials using appropriate operational rules.
\end{abstract}

Keywords: Gould-Hopper polynomials, monomiality principle, unified Apostol-type polynomials, summation formula, symmetric identity.

2020 MSC: 11B68, 33C45, 05A10.

(C)2022 All rights reserved.

\section{Introduction}

Apostol [2] introduced a class of the familiar Bernoulli numbers and polynomials when he studied the Lipschitz-Lerch Zeta maps and developed multifarious fundamental relations of these numbers and polynomials. Since Apostol's time, Apostol type numbers and polynomials in conjunction with diverse extensions have been introduced and examined by many mathematicians, for example, by Khan [7], Luo et al. [21-23], Luo [16, 17, 19], Ozarslan [24, 25], Pathan et al. [28-30], see also the references cited therein.

The Apostol-Bernoulli polynomials $B_{n}^{(\alpha)}(x ; \lambda)$, the Apostol-Euler polynomials $E_{n}^{(\alpha)}(x ; \lambda)$, and the ApostolGenocchi polynomials $G_{n}^{(\alpha)}(x ; \lambda)$ of order $\alpha \in \mathbb{C}$, are defined via the following exponential generating functions (see $[7,11-14,16,17,19,24,25,28-30])$ :

$$
\left(\frac{t}{\lambda e^{t}-1}\right)^{\alpha} e^{x t}=\sum_{n=0}^{\infty} B_{n}^{(\alpha)}(x ; \lambda) \frac{t^{n}}{n !}
$$

\footnotetext{
*Corresponding author

Email addresses: wkhan1@pmu.edu.sa (Waseem Ahmad Khan), n. sooppy@psau.edu.sa (Kottakkaran Sooppy Nisar), acikgoz@gantep.edu.tr (Mehmet Acikgoz), mtdrnugur@gmail.com \& ugur.duran@iste.edu.tr (Ugur Duran), sufianmath97@hotmail. com (Abdallah Hassan Abusufian)
}

doi: $10.22436 /$ jmcs.024.04.01

Received: 2021-01-08 Revised: 2021-01-28 Accepted: 2021-02-27 


$$
\begin{gathered}
(|t|<2 \pi \text { when } \lambda=1 ;|t|<|\log \lambda| \text { when } \lambda \neq 1) \\
\left(\frac{2}{\lambda e^{t}+1}\right)^{\alpha} e^{x t}=\sum_{n=0}^{\infty} E_{n}^{(\alpha)}(x ; \lambda) \frac{t^{n}}{n !} \\
(|t|<\pi \text { when } \lambda=1 ;|t|<|\log (-\lambda)| \text { when } \lambda \neq 1)
\end{gathered}
$$

and

$$
\begin{gathered}
\left(\frac{2 t}{\lambda e^{t}+1}\right)^{\alpha} e^{x t}=\sum_{n=0}^{\infty} G_{n}^{(\alpha)}(x ; \lambda) \frac{t^{n}}{n !} \\
(|t|<\pi \text { when } \lambda=1 ;|t|<|\log (-\lambda)| \text { when } \lambda \neq 1) .
\end{gathered}
$$

It is noted that setting $\lambda=1$ the polynomials given in (1.1) to (1.3) reduce to the classical counterparts ( $c f$. $[7,16,17,19,24,25,28-30])$ :

$$
\mathrm{B}_{\mathrm{n}}^{(\alpha)}(\mathrm{x} ; 1):=\mathrm{B}_{\mathrm{n}}^{(\alpha)}(\mathrm{x}), \mathrm{E}_{\mathrm{n}}^{(\alpha)}(\mathrm{x} ; 1):=\mathrm{E}_{\mathrm{n}}^{(\alpha)}(\mathrm{x}) \text { and } \mathrm{G}_{\mathrm{n}}^{(\alpha)}(\mathrm{x} ; 1):=\mathrm{G}_{\mathrm{n}}^{(\alpha)}(\mathrm{x})
$$

When $\alpha=1$, we obtain

$$
B_{n}^{(1)}(x ; \lambda):=B_{n}(x ; \lambda), E_{n}^{(1)}(x ; \lambda):=E_{n}(x ; \lambda) \text { and } G_{n}^{(1)}(x ; \lambda):=G_{n}(x ; \lambda),
$$

which are, respectively, classical Apostol-Bernoulli polynomials, Apostol-Euler polynomials, and the Apostol-Genocchi polynomials.

Also, in special cases,

$$
\mathrm{B}_{\mathrm{n}}^{(1)}(\mathrm{x} ; 1):=\mathrm{B}_{\mathrm{n}}(\mathrm{x}), \mathrm{E}_{\mathrm{n}}^{(1)}(\mathrm{x} ; 1):=\mathrm{E}_{\mathfrak{n}}(\mathrm{x}) \text { and } \mathrm{G}_{\mathrm{n}}^{(1)}(\mathrm{x} ; 1):=\mathrm{G}_{\mathrm{n}}(\mathrm{x})
$$

are called usual Bernoulli, Euler and Genocchi polynomials, respectively.

In recent years, a unification of the Apostol type Bernoulli, Euler, and Genocchi polynomials $Y_{n, \beta}^{(\alpha)}(x ; k, a, b)$ of order $\alpha$ are considered as follows $(c f .[14,24]$

$$
\begin{aligned}
& \left(\frac{2^{1-k^{k}}{ }^{k}}{\beta^{b} e^{t}-a^{b}}\right)^{\alpha} e^{x t}=\sum_{n=0}^{\infty} Y_{n, \beta}^{(\alpha)}(x ; k, a, b) \frac{t^{n}}{n !} \\
& \left(1^{\alpha}=1 ; k \in \mathbb{N}_{0} ; a, b \in R \backslash\{0\} ; \alpha, \beta \in \mathbb{C}\right) .
\end{aligned}
$$

One can see the reference $[14,24]$ for the details about the existence of the polynomials $Y_{n, \beta}^{(\alpha)}(x ; k, a, b)$.

Note that the polynomials $Y_{n, \beta}^{(\alpha)}(x ; k, a, b)$ include Apostol type Bernoulli, Euler, and Genocchi polynomials:

$$
Y_{n, \lambda}^{(\alpha)}(x ; 1,1,1):=B_{n}^{(\alpha)}(x ; \lambda), Y_{n, \lambda}^{(\alpha)}(x ; 0,-1,1):=E_{n}^{(\alpha)}(x ; \lambda)
$$

and

$$
Y_{n, \lambda}^{(\alpha)}(x ; 1,-1,1):=\frac{1}{2} G_{n}^{(\alpha)}(x ; \lambda) .
$$

The Appell polynomials $A_{n}(x)$ [3] for $g(t)$ are defined by the following generating function:

$$
\frac{1}{g(t)} e^{x t}=\sum_{n=0}^{\infty} A_{n}(x) \frac{t^{n}}{n !},
$$

where $g(t)$ has the following expansion:

$$
g(t)=\sum_{n=0}^{\infty} g_{n} \frac{t^{n}}{n !}, \quad g_{0} \neq 0 .
$$


The Appell class includes several significant sequences such as the Euler, Genocchi, and Bernoulli polynomials and their several generalized forms, $c f .[1-4,7-31]$ and also see the references cited therein.

The Gould Hopper polynomials $H_{n}^{(m)}(x, y)$ are defined by the following generating function [6]:

$$
e^{x t+y t^{m}}=\sum_{n=0}^{\infty} H_{n}^{(m)}(x, y) \frac{t^{n}}{n !},
$$

which are solutions of the generalized heat equation

$$
\frac{D}{D y} f(x, y)=\frac{D^{m}}{D x^{m}} f(x, y) \text { and } f(x, 0)=x^{n}
$$

Also, we note that

$$
H_{n}^{(2)}(x, y):=H_{n}(x, y) \text { and } H_{n}(2 x, 1):=H_{n}(x),
$$

where $H_{n}(x, y)$ are two variable Hermite polynomials and $H_{n}(x)$ are the classical Hermite polynomials $[1,5-7,10,24,28,34]$.

Inspired by the significance of the bivariate special functions in applications, the 2-variable general polynomials $p_{n}(x, y)$ are defined by the following exponential generating function [9]:

$$
e^{x t} \phi(y, t)=\sum_{n=0}^{\infty} p_{n}(x, y) \frac{t^{n}}{n !} \text { with } p_{0}(x, y)=1,
$$

where $\phi(y, t)$ has the following series expansion

$$
\phi(y, t)=\sum_{n=0}^{\infty} \phi_{n}(y) \frac{t^{n}}{n !} \text { with } \phi_{0}(y) \neq 0 .
$$

In view of generating function (1.6), the Gould Hopper polynomials (1.6) are the members of the 2-variable general polynomials.

The Gould-Hopper-Appell polynomials ${ }_{H} A_{n}^{(m)}(x, y)$ [4,9] (or known as the 2D Appell polynomials) and the Hermite-Appell polynomials ${ }_{H} A_{n}(x, y)$ [10], are given by the following generating functions:

$$
A(t) \exp \left(x t+y t^{m}\right)=\sum_{n=0}^{\infty} H A_{n}^{(m)}(x, y) \frac{t^{n}}{n !}
$$

and

$$
A(t) \exp \left(x t+y t^{2}\right)=\sum_{n=0}^{\infty}{ }_{H} A_{n}(x, y) \frac{t^{n}}{n !} .
$$

The polynomials $p_{n}(x, y)$ are quasi-monomial $[5,35]$ under the action of the following multiplicative and derivative operators:

$$
\widehat{M}_{p}=x+\frac{\phi^{\prime}\left(y, D_{x}\right)}{\phi\left(y, D_{x}\right)} \quad\left(D_{x}:=\frac{D}{D_{x}} \text { and } \phi^{\prime}(y, t):=\frac{D}{D_{t}} \phi(y, t)\right)
$$

and

$$
\widehat{\mathrm{P}}_{\mathrm{p}}=\mathrm{D}_{\mathrm{x}}
$$

Most of the properties of families of polynomials known as quasimonomial can be deduced by utilizing operational rules related to the appropriate derivative and multiplicative operators. The notion of quasimonomiality has been exploited within varied contexts to cope with isospectral problems [32] and 
to work the relations of new families of special functions, see [5]. According to the monomiality principle and in view of Eqs. (1.11) and (1.12), we have

$$
\widehat{M}_{p}\left\{p_{n}(x, y)\right\}=p_{n+1}(x, y) \text { and } \widehat{P}_{p}\left\{p_{n}(x, y)\right\}=n p_{n-1}(x, y) \text {. }
$$

Now since the $p_{n}(x, y)$ are quasi-monomial, the properties of these polynomials can be derived from those of the multiplicative and derivative operators $\widehat{M}_{p}$ and $\widehat{P}_{p}$ respectively. In fact, we have

$$
\widehat{M}_{p} \widehat{P}_{p}\left\{p_{n}(x, y)\right\}=n p_{n}(x, y),
$$

which gives the following differential equation satisfied by $p_{n}(x, y)$ :

$$
\left(x D_{x}+\frac{\phi^{\prime}\left(y, D_{x}\right)}{\phi\left(y, D_{x}\right)} D_{x}-n\right) p_{n}(x, y)=0 .
$$

Since $p_{0}(x, y)=1$, the $p_{n}(x, y)$ can be clearly as:

$$
p_{n}(x, y)=\widehat{M}_{p}^{n}\left\{p_{0}(x, y)\right\}=\widehat{M}_{p}^{n}\{1\},
$$

which means that the generating function of the $p_{n}(x, y)$ can be cast in the following form

$$
\exp \left(\widehat{M}_{p} t\right)\{1\}=\sum_{n=0}^{\infty} p_{n}(x, y) \frac{t^{n}}{n !},
$$

which gives the generating function (1.7). It can readily be confirmed that

$$
\left[\widehat{P}_{p}, \widehat{M}_{p}\right]=1 \text {. }
$$

For an arbitrary complex or real parameter $\lambda$ and $k \in \mathbb{N}_{0}$, the numbers $S_{k}(n, \lambda)$ is defined by means of the following exponential generating function, $c f$. [33]:

$$
\sum_{k=0}^{\infty} S_{k}(n, \lambda) \frac{t^{k}}{k !}=\frac{\lambda e^{(n+1) t}-1}{\lambda e^{t}-1}
$$

which, for $\lambda=1$, reduces to the power sum $S_{k}(n, 1):=S_{k}(n)$. Several symmetry identities for the $B_{n}^{(\alpha)}(x ; \lambda), E_{n}^{(\alpha)}(x ; \lambda)$ and $G_{n}^{(\alpha)}(x ; \lambda)$ involving a generalized sum of integer powers $S_{k}(n, \lambda)$ are derived in $[28,33]$.

In this paper, we consider unified Gould-Hopper based Apostol type polynomials and investigate some of their properties including several implicit summation formulae and some symmetric identities by series manipulation method. Moreover, we acquire several new results for unified Gould-Hopper based Apostol type polynomials by means of appropriate operational rules.

\section{On unified Gould-Hopper based Apostol type polynomials}

The generating function of the 2-variable general-Appell polynomials is provided by replacing $x$ by multiplicative operator $\widehat{M}_{p}$ of the $p_{n}(x, y)$ in (1.5):

$$
\frac{1}{g(t)} \exp \left(\widehat{M}_{p} t\right)=\frac{1}{g(t)} e^{x t} \phi(y, t)=\sum_{n=0}^{\infty} p A_{n}(x, y) \frac{t^{n}}{n !} \text {. }
$$

The polynomials ${ }_{p} A_{n}(x, y)$ are quasimonomial with respect to the following multiplicative $\widehat{M}_{p}$ and derivative $\widehat{P}_{p}$ operators (cf. [9]):

$$
\widehat{M}_{p} t=x+\frac{\phi^{\prime}\left(y, D_{x}\right)}{\phi\left(y, D_{x}\right)}-\frac{g^{\prime}\left(D_{x}\right)}{g\left(D_{x}\right)}
$$


and

$$
\widehat{P}_{p}=D_{x}
$$

In order to generate unified Gould-Hopper based Apostol type polynomials, replacing $x$ by the multiplicative operator $\widehat{M}_{p}$ in (1.4), we have

$$
\left(\frac{2^{1-k^{k}}}{\beta^{b} e^{t}-a^{b}}\right)^{\alpha} \exp \left(\widehat{M}_{p} t\right)=\sum_{n=0}^{\infty} Y_{n, \beta}^{(\alpha)}\left(\widehat{M}_{p} ; k, a, b\right) \frac{t^{n}}{n !}
$$

and

$$
\left(\frac{2^{1-k^{k}}}{\beta^{b} e^{t}-a^{b}}\right)^{\alpha} e^{x t} \phi(y, t)=\sum_{n=0}^{\infty} Y_{n, \beta}^{(\alpha)}\left(x+\frac{\phi^{\prime}\left(y, D_{x}\right)}{\phi\left(y, D_{x}\right)}-\frac{g^{\prime}\left(D_{x}\right)}{g\left(D_{x}\right)} ; k, a, b\right) \frac{t^{n}}{n !} .
$$

Thus, we give the generating function for the unified Gould-Hopper based Apostol type polynomials $p Y_{n, \beta}^{(\alpha)}(x, y ; k, a, b)$ as follows

$$
\left(\frac{2^{1-k_{t^{k}}}}{\beta^{b} e^{t}-a^{b}}\right)^{\alpha} e^{x t} \phi(y, t)=\sum_{n=0}^{\infty} p Y_{n, \beta}^{(\alpha)}(x, y ; k, a, b) \frac{t^{n}}{n !} .
$$

We remark that (2.4) and (2.5) gives the operational representation between $Y_{n, \beta}^{(\alpha)}(x ; k, a, b)$ and ${ }_{p} Y_{n, \beta}^{(\alpha)}(x, y ; k, a, b)$.

In order to frame the unified Gould-Hopper based Apostol type polynomials within the context of monomiality principle, we provide the following theorem.

Theorem 2.1. The polynomials ${ }_{p} Y_{n, \beta}^{(\alpha)}(x, y ; k, a, b)$ are quasimonomial with respect to the following multiplicative and derivative operators

$$
\widehat{M}_{p A}=x+\frac{\phi^{\prime}\left(y, D_{x}\right)}{\phi\left(y, D_{x}\right)}+\frac{\alpha k\left(\beta^{b} e^{t}-a^{b}\right)-\alpha \beta^{b} D_{x} e^{D_{x}}}{D_{x}\left(\beta^{b} e^{t}-a^{b}\right)}
$$

and

$$
\widehat{\mathrm{P}}_{\mathrm{pA}}=\mathrm{D}_{\mathrm{x}}
$$

Proof. Consider the relation

$$
D_{x}\left\{e^{x t} \phi(y, t)\right\}=t\left\{e^{x t} \phi(y, t)\right\}
$$

and differentiating (2.5) partially with respect to $t$, we find

$$
\left(x+\frac{\phi^{\prime}\left(y, D_{x}\right)}{\phi\left(y, D_{x}\right)}+\frac{\alpha k\left(\beta^{b} e^{t}-a^{b}\right)-\alpha \beta^{b} t e^{t}}{t\left(\beta^{b} e^{t}-a^{b}\right)}\right)\left\{\left(\frac{2^{1-k^{k}}}{\beta^{b} e^{t}-a^{b}}\right)^{\alpha} e^{x t} \phi(y, t)\right\}=\sum_{n=0}^{\infty} p Y_{n+1, \beta}^{(\alpha)}(x, y ; k, a, b) \frac{t^{n}}{n !} .
$$

Since $\phi(y, t)$ is an invertible series of $t$, thus $\frac{\phi^{\prime}\left(y, D_{x}\right)}{\phi\left(y, D_{x}\right)}$ has power series expansion of $t$. Hence, by (2.8), it gives

$$
\begin{gathered}
\left(x+\frac{\phi^{\prime}\left(y, D_{x}\right)}{\phi\left(y, D_{x}\right)}+\frac{\alpha k\left(\beta^{b} e^{D_{x}}-a^{b}\right)-\alpha \beta^{b} D_{x} e^{D_{x}}}{D_{x}\left(\beta^{b} e^{t}-a^{b}\right)}\right)\left\{\left(\frac{2^{1-k^{k}}}{\beta^{b} e^{t}-a^{b}}\right)^{\alpha} e^{x t} \phi(y, t)\right\} \\
=\sum_{n=0}^{\infty} p Y_{n+1, \beta}^{(\alpha)}(x, y ; k, a, b) \frac{t^{n}}{n !}
\end{gathered}
$$

which yields

$$
\sum_{n=0}^{\infty}\left(x+\frac{\phi^{\prime}\left(y, D_{x}\right)}{\phi\left(y, D_{x}\right)}+\frac{\alpha k\left(\beta^{b} e^{D_{x}}-a^{b}\right)-\alpha \beta^{b} D_{x} e^{D_{x}}}{D_{x}\left(\beta^{b} e^{t}-a^{b}\right)}\right)\left\{p Y_{n, \beta}^{(\alpha)}(x, y ; k, a, b)\right\} \frac{t^{n}}{n !}
$$




$$
=\sum_{n=0}^{\infty} p Y_{n+1, \beta}^{(\alpha)}(x, y ; k, a, b) \frac{t^{n}}{n !} .
$$

Comparing the coefficients of $\frac{\mathrm{t}^{\mathrm{n}}}{\mathrm{n} !}$ in the last equation, we get

$$
\left(x+\frac{\phi^{\prime}\left(y, D_{x}\right)}{\phi\left(y, D_{x}\right)}+\frac{\alpha k\left(\beta^{b} e^{D_{x}}-a^{b}\right)-\alpha \beta^{b} D_{x} e^{D_{x}}}{D_{x}\left(\beta^{b} e^{t}-a^{b}\right)}\right)\left\{p Y_{n, \beta}^{(\alpha)}(x, y ; k, a, b)\right\}={ }_{p} Y_{n+1, \beta}^{(\alpha)}(x, y ; k, a, b),
$$

which provides the desired result (2.6) by (1.13).

By (2.5) and (2.8), we have

$$
D_{x}\left\{\sum_{n=0}^{\infty} p Y_{n, \beta}^{(\alpha)}(x, y ; k, a, b) \frac{t^{n}}{n !}\right\}=\sum_{n=1}^{\infty} p Y_{n-1, \beta}^{(\alpha)}(x, y ; k, a, b) \frac{t^{n}}{(n-1) !},
$$

which means

$$
D_{x}\left\{p Y_{n, \beta}^{(\alpha)}(x, y ; k, a, b)\right\}=n_{p} Y_{n-1, \beta}^{(\alpha)}(x, y ; k, a, b), \quad n \geqslant 1,
$$

which gives the claimed result (2.7) via (1.13).

We give the following theorem.

Theorem 2.2. For $\mathrm{n}$ being non-negative integer, the unified Gould-Hopper based Apostol type polynomials satisfy the following differential equation

$$
\left(x D_{x}+\frac{\phi^{\prime}\left(y, D_{x}\right)}{\phi\left(y, D_{x}\right)} D_{x}+\frac{\alpha k\left(\beta^{b} e^{t}-a^{b}\right)-\alpha \beta^{b} D_{x} e^{D_{x}}}{\left(\beta^{b} e^{t}-a^{b}\right)}-n\right) p Y_{n, \beta}^{(\alpha)}(x, y ; k, a, b)=0 .
$$

Proof. Using (2.6) and (2.7) and in view of (1.15), the asserted result (2.9) can be readily obtained. So, we omit the proof.

Now, we derive some summation formulae for unified Gould-Hopper based Apostol type polynomials.

Here is the first summation formula for $p Y_{n, \beta}^{(\alpha)}(x, y ; k, a, b)$ as follows.

Theorem 2.3. The following implicit summation formula holds:

$$
p Y_{q+l, \beta}^{(\alpha)}(z, y ; k, a, b)=\sum_{n, p=0}^{q, l}\left(\begin{array}{l}
q \\
n
\end{array}\right)\left(\begin{array}{l}
l \\
r
\end{array}\right)(z-x)^{n+r}{ }_{p} Y_{q+l-r-n, \beta}^{(\alpha)}(x, y ; k, a, b) .
$$

Proof. We replace $t$ by $t+u$ and rewrite the generating function (2.5) as

$$
\left(\frac{2^{1-k}(t+u)^{k}}{\beta^{b} e^{t+u}-a^{b}}\right)^{\alpha} \phi(y, t+u)=e^{-x(t+u)} \sum_{q, l=0}^{\infty} p Y_{q+l, \beta}^{(\alpha)}(x, y ; k, a, b) \frac{t^{q}}{q !} \frac{u^{l}}{l !} .
$$

Changing $x$ by $z$ in the last equation and we can write

$$
e^{(z-x)(t+u)} \sum_{q, l=0}^{\infty} p Y_{q+l, \beta}^{(\alpha)}(x, y ; k, a, b) \frac{t^{q}}{q !} \frac{u^{l}}{l !}=\sum_{q, l=0}^{\infty} p Y_{q+l, \beta}^{(\alpha)}(z, y ; k, a, b) \frac{t^{q}}{q !} \frac{u^{l}}{l !},
$$

which gives

$$
\sum_{N=0}^{\infty} \frac{[(z-x)(t+u)]^{N}}{N !} \sum_{q, l=0}^{\infty} p_{q}^{(\alpha)} Y_{q+\beta}(x, y ; k, a, b) \frac{t^{q}}{q !} \frac{u^{l}}{l !}=\sum_{q, l=0}^{\infty} p_{q+l, \beta}^{(\alpha)}(z, y ; k, a, b) \frac{t^{q}}{q !} \frac{u^{l}}{l !}
$$


Using the following series manipulation formula

$$
\sum_{N=0}^{\infty} f(N) \frac{(x+y)^{N}}{N !}=\sum_{n, m=0}^{\infty} f(n+m) \frac{x^{n}}{n !} \frac{y^{m}}{m !}
$$

we have

$$
\sum_{n, r=0}^{\infty} \frac{(z-x)^{n+r} t^{n} u^{r}}{n ! r !} \sum_{q, l=0}^{\infty} p Y_{q+l, \beta}^{(\alpha)}(x, y ; k, a, b) \frac{t^{q}}{q !} \frac{u^{l}}{l !}=\sum_{q, l=0}^{\infty} p_{q+l, \beta}^{(\alpha)}(z, y ; k, a, b) \frac{t^{q}}{q !} \frac{u^{l}}{l !} .
$$

Now changing $q$ by $q-n, l$ by $l-p$ and utilizing the lemma [34, p.100], we get

$$
\begin{gathered}
\sum_{q, l=0}^{\infty} \sum_{n, r=0}^{q, l} \frac{(z-x)^{n+r}}{n ! r !}{ }_{p} Y_{q+l-n-r, \beta}^{(\alpha)}(x, y ; k, a, b ;) \frac{t^{q}}{(q-n) !} \frac{u^{l}}{(l-r) !} \\
=\sum_{q, l=0}^{\infty} p Y_{q+l, \beta}^{(\alpha)}(z, y ; k, a, b) \frac{t^{q}}{q !} \frac{u^{l}}{l !}
\end{gathered}
$$

which is the desired result (2.10).

Corollary 2.4. Taking $l=0$ in (2.10), we get the following result:

$$
{ }_{p} Y_{q, \beta}^{(\alpha)}(z, y ; k, a, b)=\sum_{n=0}^{q}\left(\begin{array}{l}
q \\
n
\end{array}\right)(z-x)^{n+r} Y_{q-n, \beta}^{(\alpha)}(x, y ; k, a, b) .
$$

Corollary 2.5. Replacing $z$ by $z+x$, we also obtain

$$
p \mathrm{P}_{\mathrm{q}, \beta}^{(\alpha)}(z+x, y ; k, a, b)=\sum_{n=0}^{q}\left(\begin{array}{l}
q \\
n
\end{array}\right) z_{p}^{n+r} Y_{q-n, \beta}^{(\alpha)}(x, y ; k, a, b)
$$

Theorem 2.6. The following implicit summation formula

$$
p Y_{n, \beta}^{(\alpha)}(x, y ; k, a, b)=\sum_{m=0}^{n}\left(\begin{array}{c}
n \\
m
\end{array}\right) Y_{n-m, \beta}^{(\alpha)}(k, a, b) p_{m}(x, y)
$$

is valid.

Proof. Using the definition (2.5), we have

$$
\left(\frac{2^{1-k} t^{k}}{\beta^{b} e^{t}-a^{b}}\right)^{\alpha} e^{x t} \phi(y, t)=\sum_{n=0}^{\infty} p Y_{n, \beta}^{(\alpha)}(x, y ; k, a, b) \frac{t^{n}}{n !}=\sum_{n=0}^{\infty} Y_{n, \beta}^{(\alpha)}(k, a, b) \frac{t^{n}}{n !} \sum_{m=0}^{\infty} p_{m}(x, y) \frac{t^{m}}{m !} .
$$

Using the Cauchy product and comparing the coefficients of $t^{n}$, we attain the asserted formula (2.11).

Theorem 2.7. The following summation formula holds:

$$
{ }_{p} Y_{n, \beta}^{(\alpha)}(x+z, y ; k, a, b)=\sum_{s=0}^{n}\left(\begin{array}{c}
n \\
s
\end{array}\right) z_{p}^{s} Y_{n-s, \beta}^{(\alpha)}(x, y ; k, a, b) .
$$

Proof. Replacing $y$ by $y+u$ and $x$ by $x+z$ in (2.5) and then we get

$$
\left(\frac{2^{1-k_{t}}}{\beta^{b} e^{t}-a^{b}}\right)^{\alpha} e^{(x+z) t} \phi(y, t)=\sum_{n=0}^{\infty} p Y_{n, \beta}^{(\alpha)}(x+z, y ; k, a, b) \frac{t^{n}}{n !}=\sum_{n=0}^{\infty} p Y_{n, \beta}^{(\alpha)}(x, y ; k, a, b) \frac{t^{n}}{n !} \sum_{n=0}^{\infty} \frac{z^{s} t^{s}}{s !},
$$

Utilizing the Cauchy product and comparing the coefficients of $t^{n}$, we obtain the claimed formula (2.12). 
Theorem 2.8. The following formula

$$
p Y_{n, \beta}^{(\alpha)}(y, x ; k, a, b)=\sum_{s=0}^{n}\left(\begin{array}{l}
n \\
s
\end{array}\right) Y_{n-s, \beta}^{(\alpha)}(y ; k, a, b) \phi_{s}(x)
$$

is valid.

Proof. By (2.5) to get

$$
\sum_{n=0}^{\infty} p Y_{n, \beta}^{(\alpha)}(y, x ; k, a, b) \frac{t^{n}}{n !}=\sum_{n=0}^{\infty} Y_{n, \beta}^{(\alpha)}(y ; k, a, b) \frac{t^{n}}{n !} \sum_{s=0}^{\infty} \phi_{s}(x) \frac{t^{s}}{s !} .
$$

Using the Cauchy product and comparing the coefficients of $t^{n}$, we get the desired result (2.13).

Theorem 2.9. The following implicit summation formula holds:

$$
p Y_{n, \beta}^{(\alpha)}(x-z, y ; k, a, b)=\sum_{r=0}^{n}\left(\begin{array}{c}
n \\
r
\end{array}\right) Y_{n-r, \beta}^{(\alpha)}(-z ; k, a, b) p_{r}(x, y) .
$$

Proof. From (1.7) and (2.5), we attain

$$
\left(\frac{2^{1-k_{t^{k}}}}{\beta^{b} e^{t}-a^{b}}\right)^{\alpha} e^{(x-z) t} \phi(y, t)=\sum_{n=0}^{\infty} Y_{n, \beta}^{(\alpha)}(-z ; k, a, b) \frac{t^{n}}{n !} \sum_{r=0}^{\infty} p_{r}(x, y) \frac{t^{r}}{r !},
$$

which gives

$$
\sum_{n=0}^{\infty} p Y_{n, \beta}^{(\alpha)}(x-z, y ; k, a, b) \frac{t^{n}}{n !}=\sum_{n=0}^{\infty} \sum_{r=0}^{n} Y_{n-r, \beta}^{(\alpha)}(-z ; k, a, b) p_{r}(x, y) \frac{t^{n}}{(n-r) ! r !}
$$

which means the asserted result (2.14).

Here, we give some symmetry identities for the unified Gould-Hopper based Apostol type polynomials ${ }_{p} Y_{n, \beta}^{(\alpha)}(x, y ; k, a, b)$. The results derived in this section are extensions of the previous results given by Khan [7], Ozarslan [24, 25] and Pathan and Khan [28, 30].

Theorem 2.10. The following symmetric identity

$$
\begin{aligned}
& \sum_{m=0}^{n}\left(\begin{array}{c}
n \\
m
\end{array}\right) d^{m} c^{n-m} Y_{n-m, \beta}^{(\alpha)}(d x, d y ; k, a, b)_{p} Y_{m, \beta}^{(\alpha)}(c X, c Y ; k, a, b) \\
& \quad=\sum_{m=0}^{n}\left(\begin{array}{c}
n \\
m
\end{array}\right) c^{m} d_{p}^{n-m} Y_{n-m, \beta}^{(\alpha)}(c x, c y ; k, a, b)_{p} Y_{m, \beta}^{(\alpha)}(d X, d Y ; k, a, b) .
\end{aligned}
$$

holds for $\alpha, k \in \mathbb{N}_{0} a, b \in \mathbb{R} /\{0\} ; \beta \in \mathbb{C}, x, y \in \mathbb{R}$ and $n \geqslant 0$.

Proof. By (2.5), we observe that

$$
\begin{aligned}
\Phi & =\left(\frac{c^{k} d^{k} 2^{2(1-k)} t^{2 k}}{\left(\beta^{b} e^{c t}-a^{b}\right)\left(\beta^{b} e^{d t}-a^{b}\right)}\right)^{\alpha} e^{c d x t} \phi(y, c d t) e^{c d X t} \phi(Y, c d t) \\
& =\left(\frac{c^{k} 2^{(1-k)} t^{k}}{\left(\beta^{b} e^{c t}-a^{b}\right)}\right)^{\alpha} e^{c d x t} \phi(y, c d t)\left(\frac{d^{k} 2^{(1-k)} t^{k}}{\left(\beta^{b} e^{d t}-a^{b}\right)}\right)^{\alpha} e^{c d X t} \phi(Y, c d t) \\
& =\left(\frac{c^{k} 2^{(1-k)} t^{k}}{\left(\beta^{b} e^{c t}-a^{b}\right)}\right)^{\alpha} e^{c d x t} \phi(d y, c t)\left(\frac{d^{k} 2^{(1-k)} t^{k}}{\left(\beta^{b} e^{d t}-a^{b}\right)}\right)^{\alpha} e^{c d X t} \phi(c Y, d t) .
\end{aligned}
$$


We see that the expression $\Phi$ is symmetric in $c$ and $d$. Therefore, we get

$$
\begin{aligned}
\Phi & =\sum_{n=0}^{\infty} p Y_{n, \beta}^{(\alpha)}(d x, d y ; k, a, b) \frac{(c t)^{n}}{n !} \sum_{m=0}^{\infty} p Y_{m, \beta}^{(\alpha)}(c X, c Y ; k, a, b) \frac{(d t)^{m}}{m !} \\
& =\sum_{n=0}^{\infty} \sum_{m=0}^{n} p Y_{n-m, \beta}^{(\alpha)}(d x, d y ; k, a, b) \frac{(c)^{n-m}}{(n-m) !} p Y_{m, \beta}^{(\alpha)}(c X, c Y ; k, a, b) \frac{(d)^{m}}{m !} \frac{(t)^{n}}{n !}
\end{aligned}
$$

and similarly

$$
\begin{aligned}
\Phi & =\sum_{n=0}^{\infty} p Y_{n, \beta}^{(\alpha)}(c x, c y ; k, a, b) \frac{(d t)^{n}}{n !} \sum_{m=0}^{\infty} p Y_{m, \beta}^{(\alpha)}(d X, d Y ; k, a, b) \frac{(c t)^{m}}{m !} \\
& =\sum_{n=0}^{\infty} \sum_{k=0}^{n} p Y_{n-m, \beta}^{(\alpha)}(c x, c y ; k, a, b) \frac{(d)^{n-m}}{(n-m) !} p Y_{m, \beta}^{(\alpha)}(d X, d Y ; k, a, b) \frac{(c)^{m}}{m !} \frac{(t)^{n}}{n !}
\end{aligned}
$$

which means the desired result (2.15).

Theorem 2.11. The following symmetric identity

$$
\begin{aligned}
& \sum_{m=0}^{n}\left(\begin{array}{c}
n \\
m
\end{array}\right) \sum_{i=0}^{c-1} \sum_{j=0}^{d-1} c^{n-m} d_{p}^{m} Y_{n-m, \beta}^{(\alpha)}\left(d x+\frac{d}{c} i+j, d y ; k, a, b\right) p Y_{m, \beta}^{(\alpha)}(c X, c Y ; k, a, b) \\
& =\sum_{m=0}^{n}\left(\begin{array}{c}
n \\
m
\end{array}\right) \sum_{i=0}^{d-1} \sum_{j=0}^{c-1} c^{m} d^{n-m}{ }_{p} Y_{n-m, \beta}^{(\alpha)}\left(c X+\frac{c}{d} i+j, c y ; k, a, b\right) p Y_{m, \beta}^{(\alpha)}(d X, d Y ; k, a, b)
\end{aligned}
$$

is valid for $\alpha, \mathrm{k} \in \mathbb{N}_{0} ; \mathrm{c}, \mathrm{d} \in \mathbb{R} /\{0\} ; \beta \in \mathbb{C}$ and $x, y \in \mathbb{R}$ and $\mathrm{n} \geqslant 0$.

Proof. From (2.5), we see that

$$
\begin{aligned}
\Psi & =\left(\frac{2^{2(1-k)} c^{k} d^{k} t^{2 k}}{\left(\beta^{b} e^{c t}-a^{b}\right)\left(\beta^{b} e^{d t}-a^{b}\right)}\right)^{\alpha} e^{c d x t} \phi(y, c d t) \frac{\left(e^{c d t}-1\right)^{2}}{\left(e^{c t}-1\right)\left(e^{d t}-1\right)} e^{c d X t} \phi(Y, c d t) \\
& =\left(\frac{2^{(1-k)} c^{k} t^{k}}{\left(\beta^{b} e^{c t}-a^{b}\right.}\right)^{\alpha} e^{c d x t} \phi(y, c d t)\left(\frac{e^{c d t}-1}{e^{c t}-1}\right)\left(\frac{2^{(1-k)} d^{k} t^{k}}{\left(\beta^{b} e^{d t}-a^{b}\right.}\right)^{\alpha} e^{c d X t} \phi(Y, c d t)\left(\frac{e^{c d t}-1}{e^{d t}-1}\right) \\
& =\left(\frac{2^{(1-k)} c^{k} t^{k}}{\left(\beta^{b} e^{c t}-a^{b}\right.}\right)^{\alpha} e^{c d x t} \phi(d y, c t) \sum_{i=0}^{c-1} e^{d t i}\left(\frac{2^{(1-k)} d^{k} t^{k}}{\left(\beta^{b} e^{d t}-a^{b}\right.}\right)^{\alpha} e^{c d X t} \phi(c Y, d t) \sum_{j=0}^{d-1} e^{c t j} \\
& =\sum_{i=0}^{c-1} \sum_{j=0}^{d-1}\left(\frac{2^{(1-k)} c^{k} t^{k}}{\left(\beta^{b} e^{c t}-a^{b}\right.}\right)^{\alpha} \phi(d y, c t) e^{\left(d x+\frac{d}{c} i+j\right) c t} \sum_{m=0}^{\infty} \gamma_{m, \beta}^{(\alpha)}(c X, c Y ; k, a, b) \frac{(d t)^{m}}{m !} \\
& =\sum_{n=0}^{\infty} \sum_{m=0}^{n}\left(\begin{array}{c}
n \\
m
\end{array}\right) \sum_{i=0}^{c-1} \sum_{j=0}^{d-1} c^{n-m} d_{p}^{m} Y_{n-m, \beta}^{(\alpha)}\left(d x+\frac{d}{c} i+j, d y ; k, a, b\right) p Y_{m, \beta}^{(\alpha)}(c X, c Y ; k, a, b) \frac{t^{n}}{n !} .
\end{aligned}
$$

and similarly, we get

$$
\Psi=\sum_{n=0}^{\infty} \sum_{m=0}^{n}\left(\begin{array}{l}
n \\
m
\end{array}\right) \sum_{i=0}^{d-1} \sum_{j=0}^{c-1} d^{n-m} c_{p}^{m} Y_{n-m, \beta}^{(\alpha)}\left(c x+\frac{c}{d} i+j, c y ; k, a, b\right) p Y_{m, \beta}^{(\alpha)}(d X, d Y ; k, a, b) \frac{t^{n}}{n !},
$$

which gives the desired result (2.16).

We now give another symmetric formula for unified Gould-Hopper based Apostol type polynomials as follows. 
Theorem 2.12. The following identity

$$
\begin{aligned}
& \sum_{m=0}^{n}\left(\begin{array}{c}
n \\
m
\end{array}\right) \sum_{i=0}^{c-1} \sum_{j=0}^{d-1} c^{n-m} d_{p}^{m} Y_{n-m, \beta}^{(\alpha)}\left(d x+\frac{d}{c} i, d y ; k, a, b\right) p Y_{m, \beta}^{(\alpha)}\left(c X+\frac{c}{d} j, c Y ; k, a, b\right) \\
& \quad=\sum_{m=0}^{n}\left(\begin{array}{c}
n \\
m
\end{array}\right) \sum_{i=0}^{d-1} \sum_{j=0}^{c-1} c^{m} d^{n-m} Y_{n-m, \beta}^{(\alpha)}\left(c x+\frac{c}{d} i, c y ; k, a, b\right) p Y_{m, \beta}^{(\alpha)}\left(d X+\frac{d}{c} j, d Y ; k, a, b\right)
\end{aligned}
$$

holds for each pair of integers $\mathrm{c}$ and $\mathrm{d}$ and $\mathrm{n} \geqslant 0$.

Proof. Similar to the proof of the previous theorem, we obtain

$$
\Psi=\sum_{n=0}^{\infty} \sum_{m=0}^{n}\left(\begin{array}{c}
n \\
m
\end{array}\right) \sum_{i=0}^{c-1} \sum_{j=0}^{d-1} c^{n-m} d_{p}^{m} Y_{n-m, \beta}^{(\alpha)}\left(d x+\frac{d}{c} i, d y ; k, a, b\right) p Y_{m, \beta}^{(\alpha)}\left(c X+\frac{c}{d} j, c Y ; k, a, b\right) t^{n}
$$

and

$$
\Psi=\sum_{n=0}^{\infty} \sum_{m=0}^{n}\left(\begin{array}{l}
n \\
m
\end{array}\right) \sum_{i=0}^{d-1} \sum_{j=0}^{c-1} c^{m} d^{n-m} Y_{n-m, \beta}^{(\alpha)}\left(c x+\frac{c}{d} i, c y ; k, a, b\right) p Y_{m, \beta}^{(\alpha)}\left(d X+\frac{d}{c} j, d Y ; k, a, b\right) t^{n} .
$$

which provides the asserted result (2.17).

We lastly provide the following theorem.

Theorem 2.13. The following symmetric identity

$$
\begin{aligned}
& \sum_{m=0}^{n}\left(\begin{array}{l}
n \\
m
\end{array}\right) c^{n-m} d^{m+1}{ }_{p} Y_{n-m, \beta}^{(\alpha)}(d x, d y ; k, a, b) \sum_{i=0}^{m}\left(\begin{array}{l}
m \\
i
\end{array}\right) S_{i}\left(c-1 ;\left(\frac{\beta}{a}\right)^{b}\right) p Y_{m-i, \beta}^{(\alpha)}(c X, c Y ; k, a, b) \\
& =\sum_{m=0}^{n}\left(\begin{array}{l}
n \\
m
\end{array}\right) c^{m+1} d^{n-m}{ }_{p} Y_{n-m, \beta}^{(\alpha)}(c x, c y ; k, a, b) \sum_{i=0}^{m}\left(\begin{array}{l}
m \\
i
\end{array}\right) S_{i}\left(d-1 ;\left(\frac{\beta}{a}\right)^{b}\right) p Y_{m-i, \beta}^{(\alpha)}(d X, d Y ; k, a, b)
\end{aligned}
$$

is valid for all integers $\mathrm{c}>0, \mathrm{~d}>0$ and $\mathrm{n} \geqslant 0$.

Proof. By (1.17) and (2.5), we see that

$$
\begin{aligned}
\Xi & =\frac{\left(2^{2(1-k)} c^{k} d^{k} t^{2 k}\right)^{\alpha} e^{c d x t} \phi(y, c d t)\left(\beta^{b} e^{c d t}-a^{b}\right) e^{c d X t} \phi(Y, c d t)}{\left(\beta^{b} e^{c t}-a^{b}\right)^{\alpha}\left(\beta^{b} e^{d t}-a^{b}\right)^{\alpha+1}} \\
& =\left(\frac{2^{(1-k)} c^{k} t^{k}}{\beta^{b} e^{c t}-a^{b}}\right)^{\alpha} e^{c d x t} \phi(y, c d t)\left(\frac{\beta^{b} e^{c d t}-a^{b}}{\beta^{b} e^{d t}-a^{b}}\right)\left(\frac{2^{(1-k)} d^{k} t^{k}}{\left(\beta^{b} e^{d t}-a^{b}\right.}\right)^{\alpha} e^{c d X t} \phi(Y, c d t) \\
& =\sum_{n=0}^{\infty} p Y_{n, \beta}^{(\alpha)}(d x, d y ; k, a, b) \frac{(c t)^{n}}{n !} \sum_{n=0}^{\infty} S_{n}\left(c-1 ;\left(\frac{\beta}{a}\right)^{b}\right) \frac{(d t)^{n}}{n !} \sum_{n=0}^{\infty} p Y_{n, \beta}^{(\alpha)}(c X, c Y ; k, a, b) \frac{(d t)^{n}}{n !}
\end{aligned}
$$

and similarly

$$
\Xi=\sum_{n=0}^{\infty} p Y_{n, \beta}^{(\alpha)}(c x, c y ; k, a, b) \frac{(d t)^{n}}{n !} \sum_{n=0}^{\infty} S_{n}\left(d-1 ;\left(\frac{\beta}{a}\right)^{b}\right) \frac{(c t)^{n}}{n !} \sum_{n=0}^{\infty} p Y_{n, \beta}^{(\alpha)}(d X, d Y ; k, a, b) \frac{(c t)^{n}}{n !}
$$

which yields the desired result (2.18). 


\section{Conclusions}

In the presented paper, we have considered unified Gould-Hopper based Apostol type polynomials and have investigated some of their properties including several implicit summation formulae and some symmetric identities by the series manipulation method. Moreover, we have acquired several new results for unified Gould-Hopper based Apostol type polynomials by means of appropriate operational rules.

\section{Acknowledgment}

The authors K.S. Nisar \& A.H. Abusufian thanks to the Deanship of Scientific Research (DSR), Prince Sattam bin Abdulaziz University, Saudi Arabia.

\section{References}

[1] L. C. Andrews, Special functions for engineers and mathematicians, Macmillan Co., New York, (1985). 1, 1

[2] T. M. Apostol, On the Lerch zeta function, Pacific J. Math., 1 (1951), 161-167. 1

[3] P. Appell, Sur une classe de polynômes, Ann. Sci. École Norm. Sup., 9 (1880), 119-144. 1

[4] G. Bretti, C. Cesarno, P. E. Ricci, Laguerre-type exponentials and generalized Appell polynomials, Comput. Math. Appl., 48 (2004), 833-839. 1, 1

[5] G. Dattoli, Hermite-Bessel and Laguerre-Bessel functions: a by-product of the monomiality principle, Proc. Melfi Sch. Adv. Top. Math. Phys., 1 (2000), 147-164. 1, 1, 1

[6] H. W. Gould, A. T. Hopper, Operational formulas connected with two generalization of Hermite polynomials, Duke Math. J., 29 (1962), 51-63. 1

[7] W. A. Khan, Some properties of the generalized Apostol type Hermite-based polynomials, Kyungpook Math. J., 55 (2015), 597-614. 1, 1, 1, 1, 2

[8] S. Khan, M. W. Al-Saad, R. Khan, Laguerre-based Appell polynomials: properties and applications, Math. Comput. Modelling, 52 (2010), 247-259.

[9] S. Khan, N. Raza, General-Appell polynomials within the context of monomiality principle, Int. J. Anal., 2013 (2013), 11 pages. 1, 1, 2

[10] S. Khan, G. Yasmin, R. Khan, N. A. M. Hassan, Hermite-based Appell polynomials, properties and applications, J. Math. Anal. Appl., 351 (2009), 756-764. 1, 1

[11] V. Kurt, Some symmetry identities for the unified Apostol-type polynomials and multiple power sums, Filomat, 30 (2016), 583-592. 1

[12] V. Kurt, On the unified family of generalized Apostol-type polinomials of higher order and multiple power sums, Filomat, 30 (2016), 929-935.

[13] B. Kurt, Notes on unified q-Apostol-type polynomials, Filomat, 30 (2016), 921-927.

[14] B. Kurt, S. Bilgic, Symmetry identities for the 2-variable unified Apostol-type polynomials, J. Inequal. Spec. Funct., 8 (2017), 96-103. 1, 1, 1

[15] Q.-M. Luo, Fourier expansions and integral representations for the Apostol-Bernoulli and Apostol-Euler polynomials, Math. Comp., 78 (2009), 2193-2208.

[16] Q.-M. Luo, The multiplication formulas for the Apostol-Bernoulli and Apostol-Euler polynomials of higher order, Integral Transforms Spec. Funct., 20 (2009), 377-391. 1, 1

[17] Q.-M. Luo, Some formulas for Apostol-Euler polynomials associated with Hurwitz zeta function at rational arguments, Appl. Anal. Discrete Math., 3 (2009), 336-346. 1, 1

[18] Q.-M. Luo, Fourier expansions and integral representations for the Genocchi polynomials, J. Integer Seq., 12 (2009), 1-9.

[19] Q.-M. Luo, q-extensions for the Apostol-Genocchi polynomials, Gen. Math., 17 (2009), 113-125. 1, 1

[20] Q.-M. Luo, Extensions of the Genocchi polynomials and their Fourier expansions and integral representations, Osaka J. Math., 48 (2011), 291-309.

[21] Q.-M. Luo, H. M. Srivastava,Some generalizations of the Apostol-Bernoulli and Apostol-Euler polynomials, J. Math. Anal. Appl., 308 (2005), 290-302. 1

[22] Q.-M. Luo, H. M. Srivastava, Some relationships between the Apostol-Bernoulli and Apostol-Euler polynomials, Comput. Math. Appl., 51 (2006), 631-642.

[23] Q.-M. Luo, H. M. Srivastava, Some generalizations of the Apostol-Genocchi polynomials and the Stirling numbers of the second kind, Appl. Math. Comput., 217 (2011), 5702-5728. 1

[24] M. A. Özarslan, Hermite-based unified Apostol-Bernoulli, Euler and Genocchi polynomials, Adv. Difference Equ., 2013 (2013), 13 pages. 1, 1, 1, 1, 2

[25] M. A. Özarslan, Unified Apostol-Bernoulli, Euler and Genocchi polynomials, Comput. Math. Appl., 62 (2011), $2452-$ 2462. 1, 1, 2

[26] H. Ozden, Generating function of the unified representation of the Bernoulli, Euler and Genocchi polynomials of higher order, AIP Conf. Proc., 1389 (2011), 349-352. 
[27] H. Ozden, Y. Simsek, H. M. Srivastava, A unified presentation of the generating functions of the generalized Bernoulli, Euler and Genocchi polynomials, Comput. Math. Appl., 60 (2010), 2779-2287.

[28] M. A. Pathan, W. A. Khan, Some implicit summation formulas and symmetric identities for the generalized Hermite-based polynomials, Acta Univ. Apulensis Math. Inform., 39 (2014), 113-136. 1, 1, 1, 1, 2

[29] M. A. Pathan, W. A. Khan, Some implicit summation formulas and symmetric identities for the generalized HermiteBernoulli polynomials, Mediterr. J. Math., 12 (2015), 679-695.

[30] M. A. Pathan, W. A. Khan, A new class of generalized polynomials associated with Hermite and Euler polynomials, Mediterr. J. Math., 13 (2016), 913-928. 1, 1, 2

[31] J. Sándor, B. Crstici, Handbook of number theory. II, Kluwer Academic Publishers, Dordrecht, (2004). 1

[32] Y. Smirnov, A. Turbiner, Errata: "Lie algebraic discretization of differential equations", Modern Phys. Lett. A, 10 (1995), 1795-1802. 1

[33] H. M. Srivastava, S. Araci, W. A. Khan, M. Acikgöz, A Note on the Truncated-Exponential Based Apostol-Type Polynomials, Symmetry, 11 (2019), 1-20. 1, 1

[34] H. M. Srivastava, H. L. Manocha, A treatise on generating functions, Ellis Horwood Limited. Co. New York, (1984). 1,2

[35] J. F. Steffensen, The power oid, an extension of the mathematical notion of power, Acta Math., 73 (1941), 333-366. 1 